\title{
Article \\ Effectiveness of Psychostimulant and Non-Psychostimulant Drug Therapy in the Attention Deficit Hyperactivity Disorder
}

\author{
Adriana Cojocaru ${ }^{1, \dagger}$, Lavinia Maria Hogea ${ }^{1}$, Vladimir Poroch ${ }^{2}{ }^{\oplus}$, Mihaela Adriana Simu ${ }^{1}$, \\ Virgil Radu Enatescu ${ }^{1, *}$, Roxana Jeleriu ${ }^{3,+}{ }^{,}$Nicoleta Ioana Andreescu ${ }^{3}$ (D), Maria Puiu ${ }^{3}$, \\ Bogdan Gheorghe Hogea ${ }^{4}(\mathbb{D})$, Mirela Grigoras ${ }^{5, *}$, Roxana Folescu ${ }^{5,+}$, Carmen Lăcrămioara Zamfir ${ }^{6, *(D)}$, \\ Ileana Enatescu ${ }^{7}$ and Laura Alexandra Nussbaum ${ }^{1}$
}

check for updates

Citation: Cojocaru, A.; Hogea, L.M.; Poroch, V.; Simu, M.A.; Enatescu, V.R.; Jeleriu, R.; Andreescu, N.I.; Puiu, M.; Hogea, B.G.; Grigoras, M.; et al.

Effectiveness of Psychostimulant and Non-Psychostimulant Drug Therapy in the Attention Deficit Hyperactivity Disorder. Appl. Sci. 2021, 11, 502. https://doi.org/10.3390/app11020502

Received: 3 December 2020

Accepted: 3 January 2021

Published: 6 January 2021

Publisher's Note: MDPI stays neutral with regard to jurisdictional clai$\mathrm{ms}$ in published maps and institutional affiliations.

Copyright: $(\odot 2021$ by the authors. Licensee MDPI, Basel, Switzerland. This article is an open access article distributed under the terms and conditions of the Creative Commons Attribution (CC BY) license (https:// creativecommons.org/licenses/by/ $4.0 /)$.
1 Department of Neuroscience, “Victor Babes" University of Medicine and Pharmacy, 2 Eftimie Murgu Square, 300041 Timisoara, Romania; cojocaru.adriana@umft.ro (A.C.); hogea.lavinia@umft.ro (L.M.H.); simu.mihaela@umft.ro (M.A.S.); nussbaum.laura@umft.ro (L.A.N.)

2 2nd Department of Internal Medicine, Grigore T. Popa University of Medicine and Pharmacy, 16 Universitatii Str., 700115 Iasi, Romania; vladimir.poroch@umfiasi.ro

3 Department of Microscopic Morphology, Victor Babes" University of Medicine and Pharmacy, 2 Eftimie Murgu Square, 300041 Timisoara, Romania; jeleriu.roxana@umft.ro (R.J.); andreescu.nicoleta@umft.ro (N.I.A.); puiu.maria@umft.ro (M.P.)

4 Department of Orthopedics and Trauma, "Victor Babes" University of Medicine and Pharmacy, 2 Eftimie Murgu Square, 300041 Timisoara, Romania; hogea.bogdan@umft.ro

5 Department of Anatomy and Embryology, "Victor Babes" University of Medicine and Pharmacy, 2 Eftimie Murgu Square, 300041 Timisoara, Romania; folescu.roxana@umft.ro

6 Department of Morpho-Functional Sciences I, “Grigore T Popa” University of Medicine and Pharmacy, 16 Universitatii str, 700115 Iasi, Romania

7 Department of Obstetrics-Gynecology, "Victor Babes” University of Medicine and Pharmacy, 2 Eftimie Murgu Square, 300041 Timisoara, Romania; enatescu.ileana@umft.ro

* Correspondence: enatescu.virgil@umft.ro (V.R.E.); grigoras.mirela@umft.ro (M.G.); carmen.zamfir@umfiasi.ro (C.L.Z.)

+ These authors have equal contribution.

Abstract: Attention Deficit Hyperkinetic Disorder (ADHD) is a neurobiological behavioral disorder of the child, adolescent, and adult characterized by problems of concentration, hyperactivity, and impulsivity caused by an imbalance of chemical neurotransmitters in the brain-dopamine and noradrenaline. ADHD first-line drugs are divided in psychostimulant, as Methylphenidate and Amphetamines and non-psychostimulant medications-Atomoxetine (the only representative nonpsychostimulant medication approved in our country in children and adolescents). The purpose of our research was to assess the clinical evolution of patients with ADHD based on the drug treatment that is administered: psychostimulant or non-psychostimulant. Both psychostimulantMethylphenidate, and non-psychostimulant therapy-Atomoxetine, proved to significantly improve the symptoms of attention deficit hyperkinetic disorder. There was a significant reduction in the severity of ADHD symptoms at six months and at one year from the start of treatment in the case of the psychostimulant group, whereas in the non-psychostimulant group, the significant reduction in severity of symptomatology occurs only at six months after the start of treatment. We can conclude that both types of drugs are effective in reducing the severity of symptoms and in improving the clinical condition of patients with ADHD, but the comparative analysis of the two groups demonstrated that significantly better results are obtained with psychostimulant treatment.

Keywords: psychostimulants; non-psychostimulants; ADHD; methylphenidate; Atomoxetine; clinical condition

\section{Introduction}

Attention Deficit Hyperkinetic Disorder (ADHD) is a neurobiological behavioural disorder of the child, adolescent, and adult characterized by problems of concentration, 
hyperactivity, and impulsivity caused by an imbalance of chemical neurotransmitters in the brain-dopamine and noradrenaline, affecting those parts of the brain responsible for self-monitoring and for suppressing inappropriate behaviours [1-3].

At present, ADHD is a major public health problem due to its high prevalence. Currently, it is considered the most common neuropsychiatric disorder, affecting $5-10 \%$ of children and adolescents [2,4-6].

There are theories that involve the effect of dopamine and noradrenaline neurotransmitters in the onset of ADHD. The response of patients with ADHD to psychostimulants, which facilitate dopamine release, has led to the speculation that, in some areas of the brain, these neurotransmitters have deficiencies in the transmission of nerve impulses. In children diagnosed with ADHD, imaging investigations revealed a decrease in cerebral activity in the frontal and parietal cortex and in cerebellum [6-9].

The diagnosis of ADHD requires both developmentally inappropriate symptoms of inattention and/or hyperactivity and impulsivity and functional impairments in multiple settings. Functional domains affected by ADHD include academic functioning, peer relationships, and family functioning. Usually, functional impairments, rather than emerging symptoms, tend to be the primary reason for clinical referral [4-6].

The approach to the ADHD is multimodal, involving also drug therapies. Nowadays, the first-line drugs used to treat ADHD are divided in two major classes: psychostimulant and non-psychostimulant medications $[6,10]$.

Psychostimulants correct dopamine deficits in the central nervous system by blocking dopamine uptake mechanisms at this level in increasing the extracellular level of dopamine. There are studies that have shown that psychostimulants gradually regulate the functioning of neurons in the prefrontal cortex. The most commonly used drugs in this class are methylphenidate and amphetamines $[1,2,5,6]$.

Non-psychostimulant medications have a different mechanism to increase free norepinephrine. They make a blockage in the neuronal synapse, resulting in a relative increase of noradrenaline at this level. The benefits of non-psychostimulant medications consist in a long time action, facility of administration, requiring a single dose per day, the absence of rebound phenomena, low risk of abuse, and flexibility in dose adjustment. Atomoxetine is the only representative of this class approved in our country in children and adolescents $[2,3,6]$.

The purpose of our research was to track the clinical evolution of patients with ADHD based on the drug treatment that is administered: psychostimulant or non-psychostimulant.

\section{Materials and Methods}

The present research was performed between the years 2012 and 2019, in the University Hospital for Child and Adolescent Psychiatry and Neurology Timișoara.

The study was conducted on a total of 80 patients diagnosed with ADHD, the combined subtype, aged between 7 and 18 years, admitted in the Child and Adolescent Psychiatry Clinic, during the period 2012-2019.

We obtained for each patient the informed assent and the informed consent from the parents/legal guardians. Our study was done in accordance with the Ethical Committee regulations of the "Victor Babes" University of Medicine and Pharmacy, Timișoara and with the ICH-GCP (International Conference on Harmonisation-Good Clinical Practice) regulations and guidelines.

Patients were diagnosed with the combined subtype of ADHD according to DSM-IVTR (Diagnostic and Statistical Manual of Mental Disorders IV.-Text Revised) and ICD-10 (International Classification of Diseases-10) criteria. The clinical evolution of each patient was analysed over a two-year period, depending on the type of medication administeredpsychostimulant or non-psychostimulant.

The 80 patients enrolled in the study were divided in two groups, the distribution criterion being the type of medication administered: psychostimulant or non-psychostimulant. The patients were randomly assigned in the two groups of treatment. Each group consisted 
of 40 patients diagnosed with the combined type of ADHD. Subjects of group 1 received psychostimulant medication-Methylphenidate, while subjects of group 2 received nonpsychostimulant medication-Atomoxetine.

The comparative demographic data of the two studied groups is captured in Table 1.

Table 1. Demographic data of the studied ADHD groups.

\begin{tabular}{ccccc}
\hline \multicolumn{2}{c}{ Analysed Variable } & Group 1 & Group 2 & $p$ \\
\hline \multirow{2}{*}{ Age } & $7-12$ years & $41 \%$ & $43 \%$ & $>0.05$ \\
\cline { 2 - 5 } & $12-18$ years & $59 \%$ & $57 \%$ & $>0.05$ \\
\hline \multirow{2}{*}{ Gender } & Girls & $34 \%$ & $37 \%$ & $>0.05$ \\
\cline { 2 - 5 } & Boys & $66 \%$ & $63 \%$ & $>0.05$ \\
\hline
\end{tabular}

\subsection{Demographic Data of the Studied ADHD Groups}

Methylphenidate was administered orally once daily in the morning; we began with low doses-18 $\mathrm{mg}$ - and increased them at weekly intervals with $18 \mathrm{mg}$ at a maximum of $54 \mathrm{mg}$ for patients who have not achieved an optimal response at a lower dose. The optimal dosage was calculated also in function of the weight, age, and the intensity of symptoms of the patients. In our country, the extended-release Methylphenidate tablets were available in $18 \mathrm{mg}, 36 \mathrm{mg}$, and $54 \mathrm{mg}$ dosage strengths.

Atomoxetine was administered in an initial dose of $0.5 \mathrm{mg} / \mathrm{kg} /$ day orally in the morning and after seven days, increasing to the maintenance dose of $1.2 \mathrm{mg} / \mathrm{kg} /$ day. Atomoxetine tablets being available in $10 \mathrm{mg}, 18 \mathrm{mg}, 25 \mathrm{mg}, 40 \mathrm{mg}, 60 \mathrm{mg}$, and $80 \mathrm{mg}$ dosage strengths.

All patients enrolled in the study were assessed using the following scales: ADHD Rating Scale, Children's Global Assessment Scale (CGAS), Clinical Global Impression Severity/Improvement Scale/ (CGI-S/CGI-I).

To assess the efficacy of the drug therapy with the ADHD Rating Scale, mean total baseline scores were compared with the scores at six months, one year, and two years after the start of the treatment.

Clinical improvement of symptomatology was assessed using the CGI-scale, the component of clinical improvement assessment.

The assessment of the evolution of the overall functioning of children was done using the Children's Global Assessment Scale (CGAS).

\subsection{Statistical Analysis}

All analyses were carried out using SPSS software (version 17.0, Chicago, IL, USA) and Microsoft Excel. For comparing the clinical scales scores (ADHD-RS, CGI-S/I, CGAS), the Friedman nonparametric test for pair values was used. For comparing the clinical response, evolution between the groups-G1 (patients with psychostimulant treatment) and the group G2 (patients with non-psycho-stimulant treatment), the Mann-Whitney non-parametric test was applied. For comparing the mean total clinical scales scores at two different time points and in each two with two different time points, the nonparametric test Wilcoxon signed Ranks was used. The data are presented as mean and/or standard deviation. Depending on the variables processed to evaluate statistical significance, we used unpaired Student $t$-tests and $t$ student tests for dependent samples. The condition that they be statistically significant is that the result should be $p<0.05$.

\section{Results}

We assessed the efficacy of the drug treatment, following the evolution of the scores obtained on the ADHD-RS scale, in correlation with the psychostimulant or non-psychostimulant medication administered (Table 2). 
Table 2. Psychostimulant and non-psychostimulant medication for ADHD.

\begin{tabular}{ccc}
\hline ADHD Medication & Psychostimulant & Non-psychostimulant \\
\hline Methylphenidate & +++ & \\
Amphetamines & +++ & +++ \\
Atomoxetine & & + \\
\hline
\end{tabular}

\subsection{Psychostimulant and Non-Psychostimulant Medication for ADHD}

Table 3 shows the comparative analysis of the evolution of the symptomatology frequency in the two groups, according to the type of medication administered, through the total mean scores obtained on the ADHD-RS scale by the subjects of group 1 versus group 2.

Table 3. Comparative analysis of the ADHD-RS scores between group 1 with psychostimulants and group 2 with nonpsychostimulants.

\begin{tabular}{cccccccccc}
\hline ADHD Rating Scale & \multicolumn{3}{c}{$\begin{array}{c}\text { Mean Total Scores Obtained in } \\
\text { G1-Psychostimulants }\end{array}$} & \multicolumn{3}{c}{$\begin{array}{c}\text { Mean Total Scores Obtained in } \\
\text { G2-Non-Psychostimulants }\end{array}$} \\
\hline Time Point & Mean & $\begin{array}{c}\text { Std. Devi- } \\
\text { ation }\end{array}$ & Min. & Max. & Mean & $\begin{array}{c}\text { Std. Devi- } \\
\text { ation }\end{array}$ & Min. & Max. & Sign \\
\hline BASELINE & 41 & 2.92 & 16.00 & 25.00 & 39 & 2.11 & 15.00 & 23.00 & - \\
6 Months & 30 & 2.78 & 17.40 & 27.70 & 34 & 1.42 & 27.40 & 37.80 & 0.046 \\
1 Year & 25 & 1.93 & 23.80 & 30.00 & 30 & 1.20 & 17.40 & 27.78 & 0.007 \\
2 Years & 15 & 2.14 & 24.50 & 33.00 & 25 & 1.78 & 24.39 & 33.78 & 0.0001 \\
\hline
\end{tabular}

3.2. Comparative Analysis of the ADHD-RS Scores between Group 1 with Psychostimulants and Group 2 with Non-Psychostimulants

We compared the total mean scores obtained at baseline on the ADHD-RS scale by the patients in the two groups with those obtained at six months, one year, and two years after treatment (psychostimulant with Methylphenidate or non-psychostimulant with Atomoxetine). We have noticed that a significant reduction in overall mean scores has occurred in both groups. However, we found that total mean scores were significantly more reduced in the group treated with Psychostimulants. $p<0.05$ was present in all three assessments performed since the start of treatment.

The significant reduction in the frequency of ADHD symptoms is observed even six months after the treatment. The scores continue to decline significantly also at one and respectively, two years, after the treatment. In the non-psychostimulant treatment group (Atomoxetine), too, there was a significant reduction in total mean scores on the ADHD-RS from baseline to six months, one year, and two years after the start of treatment.

Through the assessment of the efficacy of the drug treatment following the evolution over time of the scores obtained on the CGI-S scale, the statistical analysis did not reveal a significant difference between the total mean scores obtained on the CGI-S scale of the two groups ( $p$ greater than 0.05). However, it can be observed that the tendency to reduce the scores is higher in the group treated with psychostimulants. In this group, the scores dropped at six months and at one year post-treatment, while in group 2, the scores decreased only at six months after the treatment started.

3.3. Comparative Analysis between Group 1 with Psychostimulants and Group 2, Concerning the CGI-S Scale Scores, in Different Time Points

The severity assessment component of the CGI-S scale evaluates the degree of severity of the symptoms caused by ADHD (Table 4). 
Table 4. Comparative analysis between group 1 with psychostimulants and group 2, concerning the Clinical Global Impression Severity (CGI-S) scale scores, in different time points.

\begin{tabular}{cccc}
\hline \multirow{2}{*}{ CGI-Severity } & Mean Scores Group & Mean Scores Group & $p$ \\
\hline Baseline & $\mathbf{1}$ & $\mathbf{2}$ & $\mathbf{0 . 5}$ \\
6 months & 5 & 5 & $\mathbf{1}$ \\
1 year & 4 & 4 & $\mathbf{0 . 2 3}$ \\
2 years & 3 & 4 & $\mathbf{0 . 2 3}$ \\
\hline
\end{tabular}

In the group treated with psychostimulants-Methylphenidate, we noted that there was a significant reduction in 6-month and respectively, one year scores $(p=0.049)$, after treatment $(p=0.049)$, followed by a plateau phase in which the total mean scores remain unchanged. Thus, in this group, the total scores range from 5 (marked effect) to 4 (moderate effect) after six months, and 3 (slight effect) after one year. At two years the average of total scores remains unchanged.

In the group treated with Atomoxetine, the mean of total score starts also from 5 (the patient is severely affected) and there is a significant change at six months after treatment, reaching a mean of total scores of 4 (moderate). There is no improvement at one year or two years after the treatment, the mean of the scores being still 4 .

Through the comparative analysis of the scores obtained by group 1 and group 2 on the CGI-I scale, we obtained the following results: there were no statistically significant differences between the two groups in terms of scores obtained on the CGI-I scale, the component that assesses the patients' clinical improvement status $(p>0.5)$. Thus, both types of treatment psychostimulant with Methylphenidate and non-psychostimulant with Atomoxetine are effective in improving ADHD.

3.4. Comparative Analysis between Group 1 with Psychostimulants and Group 2, Concerning the CGAS Scale Scores, in Different Time Points

We also assessed the efficacy of drug therapy following the evolution over time of the scores obtained on the CGAS (Table 5).

Table 5. Comparative analysis between group 1 with psychostimulants and group 2, concerning the Children's Global Assessment Scale (CGAS) scale scores, in different time points.

\begin{tabular}{cccccccccc}
\hline $\begin{array}{c}\text { ADHD } \\
\text { Rating Scale }\end{array}$ & \multicolumn{3}{c}{$\begin{array}{c}\text { Mean Total Scores Obtained in } \\
\text { G1-Psychostimulants }\end{array}$} & \multicolumn{3}{c}{$\begin{array}{c}\text { Mean Total Scores Obtained in } \\
\text { G2-Non-Psychostimulants }\end{array}$} \\
\hline Time Point & Mean & $\begin{array}{c}\text { Std. Devi- } \\
\text { ation }\end{array}$ & Min. & Max. & Mean & $\begin{array}{c}\text { Std. Devi- } \\
\text { ation }\end{array}$ & Min. & Max. & Sign \\
\hline BASELINE & $38 \%$ & 2.32 & 33.00 & 43.00 & $39 \%$ & 2.31 & 35.00 & 46.00 & 0.31 \\
6 Months & $65 \%$ & 2.58 & 60.40 & 69.70 & $56 \%$ & 1.47 & 52.40 & 60.80 & 0.000062 \\
1 Year & $73 \%$ & 1.96 & 70.80 & 78.00 & $70 \%$ & 1.28 & 60.40 & 74.00 & 0.08 \\
2 Years & $80 \%$ & 2.12 & 74.50 & 87.40 & $71 \%$ & 1.85 & 64.39 & 77.78 & 0.000062 \\
\hline
\end{tabular}

In the group treated with psychostimulants-Methylphenidate, the test started at an operating level of $38 \%$. After the beginning of the intervention, an extremely significant increase in functional level was observed at six months $(p<0.01)$, reaching an average of $65 \%$. Moreover, one year after the treatment administration, there was a significant increase in the level of functioning, reaching an average of $73 \%(p=0.011)$. At two years, the level of operation reached $80 \%$.

In the group treated with non-psychostimulants-Atomoxetine, the test started at a level of 39\%. After treatment administration, there was a significant increase in the level of functioning $(p<0.05)$ reaching $56 \%$ after six months. One year after treatment, the increase in the level of functioning was still significant $(p<0.05)$, reaching $70 \%$. 
The statistical analysis revealed significant differences between the two groups in terms of increasing the level of global functioning $(p<0.05)$. In the group treated with psychostimulant agents, the level of overall functioning increased significantly more than in the group receiving non-psychostimulant medication especially at six months and at two years from the start of treatment administration.

Using the total mean scores obtained on the ADHD-RS, we quantified the efficacy of the drug treatment in reducing the symptoms of ADHD under treatment. We observed that in the group treated with psychostimulants but also in that treated with non-psychostimulants, symptom relief was significant $(p<0.05)$. However, the statistical analysis revealed that the symptoms were significantly reduced in the psychostimulanttreated group in all three post-treatment evaluations.

In the group treated with psychostimulants, we noted that there was a significant reduction in the 6-month and respectively, one year scores $(p=0.049)$, after treatment $(p=0.49)$, followed by a plateau phase, in which the total mean scores remain unchanged. Thus, in this group, the mean of total scores ranges from 5 (markedly ill) to 4 (moderately ill) after six months, and 3 (mildly ill) after one year. In the group treated with Atomoxetine, the mean of total scores starts also at 5 (the patient is markedly ill) and there is a significant change at only six months after treatment, reaching a mean value of 4 (moderately ill). However, on the graphical representation it was observed that the tendency to reduce the scores is higher in the group treated with psychostimulants. In this group, the scores dropped at just six months and at one year post-treatment, while in group 2, the scores decreased only six months after the treatment started.

The statistical analysis revealed significant differences between the two groups in terms of increasing the level of global functioning $(p<0.05)$. In the group treated with psychostimulant agents, the level of overall functioning increased significantly more than in the group receiving non-psychostimulant medications at six months and two years from the start of treatment. At one year, the differences were statistically insignificant $(p=0.08)$.

\section{Discussion}

We have noticed that a significant reduction in overall mean scores has occurred in both groups, but like in some other studies, we found that total mean scores were significantly more reduced in the group treated with psychostimulants [6].

In the group treated with psychostimulants-Methylphenidate, we noted that there was a significant reduction in symptoms after six months and one year, after treatment, followed by a plateau phase in which the total mean scores remain unchanged.

In the group treated with Atomoxetine, there was no improvement at one year or two years after the treatment, the mean values of improvement of symptoms being still the same.

Thus, both types of treatment, psychostimulant with Methylphenidate and nonpsychostimulant with Atomoxetine, are effective in improving ADHD [6].

In the group treated with psychostimulant agents, the level of overall functioning increased significantly more than in the group receiving non-psychostimulant medication especially at six months and at two years from the start of treatment administration [10-12].

Today, the medical treatment of ADHD is represented by psychostimulant-Methylphenidate, and non-psychostimulant drugs-Atomoxetine [6,10-12]. In the absence of adequate treatment, ADHD has an unfavourable prognosis and may result in complications.

Thus, it can be said that both types of treatment are effective in reducing the aggressiveness of ADHD symptoms, but psychostimulants bring improvements faster and over a longer period of time [6,12].

Therefore, psychostimulants-Methylphenidate, are found to be more effective than non-psychostimulants in ADHD treatment [6,10-12].

Most studies conducted so far suggest that both psychostimulants and non-psychostimulants are effective in treating ADHD. However, it has been found that better and faster results are obtained with psychostimulating therapy [12]. 
In a study conducted in 2010, Antonucci et al. found that the most important improvements observed after administration of psychostimulants refer to the main symptoms of the disorder. Improvements in executive functions, interpersonal relationships, as well as reduced association of behavioural disorders in children with ADHD have also been observed [6,12].

At the same time, in an open-label study in 2006, Kratochvil et al. suggest that Atomoxetine is as effective as Methylphenidate in treating ADHD, both drugs having very good effects in alleviating attention deficit and hyperactivity/impulsivity [13].

In 2003, Michelson et al. performed eight control studies and six open-label studies to assess the efficacy of Atomoxetine in ADHD patients. An initial 10-week study on ADHD subjects revealed a $30 \%$ reduction in total mean scores on Conners Scale, after Atomoxetine administration, compared to $20 \%$ representing the drop of total mean scores when a placebo drug was administered. Similar results have been obtained in the case of reduction of the symptoms of inattention and hyperactivity/impulsivity [14].

Another study, performed by Timothy Wilens et al., showed the fact, that psychostimulants reduce ADHD symptoms in 70\% of the children and adolescents with this diagnosis. At the same time, the study proved that the administration of psychostimulants in childhood determines the reduction of risk of comorbidities onset during adolescence [15].

In another study, performed in 2010 by Marchant et al., during a six months period, it was observed that for $85 \%$ of the included subjects, the CGI-I scale scores improved after the administration of Methylphenidate. Moreover, the registered adverse events were minimal [16].

In 2010 Hellwig et al. publish a study performed with 67 male subjects, aged between 6 and 13 years, diagnosed with ADHD. They observed through the study that the subjects treated with Methylphenidate had a higher level of language comprehension on the WISCIV scale (Wechsler Intelligence Scale for Children-IV), in comparison with the subjects, who did not receive psychostimulant treatment [17].

\section{Conclusions}

Both psychostimulant-Methylphenidate, and non-psychostimulant therapy-Atomoxetine, significantly improve the symptoms of attention deficit hyperactivity disorder. Although both therapies have been found to be effective in reducing the frequency of ADHD symptoms, our research has shown that psychostimulants significantly reduce the symptoms of ADHD. There was a significant reduction in the severity of ADHD symptoms at six months and at one year from the start of treatment in the case of the psychostimulant group, whereas in the non-psychostimulant group, the significant reduction in severity of symptomatology occurs only at six months after the start of treatment.

We can conclude, that both types of drugs are effective in reducing the severity of symptoms and in improving the clinical condition of patients with ADHD.

Both psychostimulant and non-psychostimulant therapy significantly improve the overall functioning of patients with ADHD, but our research demonstrated that significantly better results are obtained with psychostimulant treatment.

Author Contributions: A.C., L.A.N., L.M.H., M.A.S.—conceptualization and investigation; V.R.E., I.E., M.A.S., N.I.A., R.J. and M.P.- data curation and methodology; V.P., B.G.H., M.G. and R.F. for writing the original draft, C.L.Z.- review and editing. All authors have read and agreed to the published version of the manuscript.

Funding: This research received no external funding.

Informed Consent Statement: Informed consent was obtained from all subjects involved in the study.

Data Availability Statement: Not applicable.

Conflicts of Interest: The authors declare no conflict of interest. 


\section{References}

1. Conners, C.K. Attention-deficit/hyperactivity disorder: Historical development and overview. J. Atten. Disord. $2000,3,173-191$.

2. Biederman, J.; Faraone, S.V.; Keenan, K.; Knee, E. Family-genetic and psychosocial risk factors in DSM-III attention deficit disorder. J. Am. Acad. Child. Adolesc. Psychiatry 1990, 29, 526-533. [CrossRef] [PubMed]

3. Malenka, R.C.; Nestler, E.J.; Hyman, S.E. Molecular Neuropharmacology: A Foundation for Clinical Neuroscience, 2nd ed.; McGraw-Hill Medical: New York, NY, USA, 2009; pp. 154-197.

4. Dulcan, M.K.; Lake, M.B. Concise Guide to Child and Adolescent Psychiatry, 4th ed.; American Psychiatric Publishing: New York, NY, USA, 2011; pp. 128-146.

5. Gentile, J.P.; Atiq, R.; Gillig, P.M. Adult ADHD: Diagnosis, Differential Diagnosis, and Medication Management-Psychiatry, 2nd ed.; Edgmont: New York, NY, USA, 2006; pp. 59-78.

6. Robe, A.; Dobrean, A.; Cristea, I.A.; Păsărelu, C.R.; Predescu, E. Attention-deficit/hyperactivity disorder and task-related heart rate variability: A systematic review and meta-analysis. Neurosci. Biobehav. Rev. 2019, 99, 11-22. [CrossRef] [PubMed]

7. Andreescu, N.I.; Cosma, M.; Farcas, S.S.; Stian, M.; Amzar, D.G.; Puiu, M. Assessment of chromosomal aneuploidies in sperm of infertile males by using FISH technique. Rom. J. Morphol. Embryol. 2016, 57, 173-178. [PubMed]

8. Nussbaum, L.; Gradinaru, R.; Andreescu, N.; Dumitrascu, V.; Tudor, A.; Suciu, L.; Stefanescu, R.; Puiu, M. The response to atypical antipsychotic drugs in correlation with the CYP2D6 genotype: Clinical implications and perspectives. Farmacia 2014, 62, 1191-1201.

9. Prince, J.B. Pharmacotherapy of attention-deficit hyperactivity disorder in children and adolescents: Update on new stimulant preparations, atomoxetine, and novel treatments. Child. Adolesc. Psychiatr. Clin. N. Am. 2006, 15, 13-50. [CrossRef]

10. Ageu, L.S.; Levai, C.M.; Andreescu, N.I.; Grigoras, M.L.; Hogea, L.M.; Chiriac, D.V.; Folescu, R.; Bredicean, A.C.; Nussbaum, L.M.; Enstescu, V.R. Modern molecular study of weight gain related to antidepressant treatment: Clinical implications of the pharmacogenetic testing. Rom. J. Morphol. Embryol. 2018, 59, 165-173. [PubMed]

11. Nussbaum, L.A.; Hogea, L.M.; Chiriac, D.V.; Grigoras, M.L.; Folescu, R.; Bredicean, A.C.; Rosca, E.C.I.; Muncan, B.; Nussbaum, L.M.; Simu, M.A.; et al. The effect of neurobiological changes in the brain of children with schizophrenia, ultra high-risk for psychosis and epilepsy: Clinical correlations with EEG and neuroimagistic abnormalities. Rom. J. Morphol. Embryol. 2017, 58, 1435-1446.

12. Antonucci, D.; Kunins, C.; Manos, M.; Lopez, F.A.; Kerney, D.L. Assessing effects of treatment with lisdexamfetamine dimesylate for pediatric ADHD using a parental survey. CNS Spectr. 2010, 15, 248-256. [CrossRef] [PubMed]

13. Kratochvil, C.J.; Wilens, T.E.; Greenhill, L.L.; Gao, H.; Baker, K.D.; Feldman, P.D.; Gelowitz, D.L. Effects of Long-Term Atomoxetine Treatment for Young Children With Attention-Deficit/Hyperactivity Disorder. J. Am. Acad. Child. Adolesc. Psychiatry 2006, 45, 919-927. [CrossRef] [PubMed]

14. Michelson, D.; Adler, L.; Spencer, T.; Reimherr, F.W.; West, S.A.; Allen, A.J. Atomoxetine in adults with ADHD: two randomized, placebo-controlled studies. Biol. Psychiatry 2003, 53, 112-120. [CrossRef]

15. Wilens, T.E.; Spencer, T.J. Understanding attention-deficit/hyperactivity disorder from childhood to adulthood. Postgrad Med. 2010, 122, 97-109. [CrossRef] [PubMed]

16. Marchant, B.K.; Reimherr, F.W.; Halls, C.; Williams, E.D.; Strong, R.E. OROS methylphenidate in the treatment of adults with ADHD: A 6-month, open-label, follow-up study. Ann. Clin. Psychiatry 2010, 22, 196-204. [PubMed]

17. Hellwig-Brida, S.; Daseking, M.; Keller, F.; Petermann, F.; Goldbeck, L. Effects of methylphenidate on intelligence and attention components in boys with attention-deficit/hyperactivity disorder. J. Child. Adolesc Psychopharmacol. 2011, 21, 245-253. [CrossRef] [PubMed] 